$\operatorname{IPPP} / 01 / 09$

$\mathrm{DCPT} / 01 / 18$

20 February 2001

\title{
Rates for rapidity gap Higgs production
}

\author{
V.A. Khoze ${ }^{a}$, A.D. Martin $^{a}$ and M.G. Ryskin ${ }^{a, b}$ \\ ${ }^{a}$ Department of Physics and Institute for Particle Physics Phenomenology, University of \\ Durham, Durham, DH1 3LE \\ ${ }^{b}$ Petersburg Nuclear Physics Institute, Gatchina, St. Petersburg, 188300, Russia
}

\begin{abstract}
We present model-independent estimates of the signal-to-background ratio for Higgs $\rightarrow b \bar{b}$ detection in double-diffractive events at the Tevatron and the LHC. For the missingmass approach to be able to identify the Higgs boson, it will be necessary to tag the $b$ quark jets in the central region. The signal is predicted to be very small at the Tevatron, but observable at the LHC. We note that the double-diffractive dijet production, may serve as a unique gluon factory. This process can be used also as a Pomeron-Pomeron luminosity monitor.
\end{abstract}

\footnotetext{
${ }^{\dagger}$ Based on a talk by V.A. Khoze at the First Workshop on Forward Physics and Luminosity Determination at LHC, Helsinki, 31 October - 4 November, 2000.
} 


\section{Introduction}

It looks quite appealing to study central production processes with a large rapidity gap on either side in high energy hadron collisions. These embrace double-diffractive reactions generated by 'Pomeron-Pomeron' fusion, searches for new objects (such as the Higgs boson), and new approaches to study conventional physics, including the investigation of subtle aspects of QCD. Equally interesting are other processes mediated by the colourless $t$-channel exchanges, especially the $W$-boson fusion reactions.

One possibility, which at first sight looks attractive, is the search for the Higgs boson in double rapidity gap events at proton colliders, see, for example, [1]-[9]. This has become more important since LEP is no longer available for Higgs hunting. An obvious advantage of the rapidity gap approach is the spectacularly clean experimental signatures (hadron-free zones between the remnants of the incoming protons and the produced system) and the possibility to clearly differentiate between different production mechanisms.

Events with large rapidity gaps may be selected either by using a calorimeter or by detecting leading protons with beam momentum fractions $x_{p}$ close to 1 . If the momenta of the leading protons can be measured with very high precision then a centrally produced state may be observed as a peak in the spectrum of the missing-mass $(M)$ distribution. Indeed, it has recently been proposed [10] to supplement CDF with very forward detectors to measure both proton and antiproton in Run II of the Tevatron in events with the fractional momentum loss $\xi=1-x_{p} \lesssim 0.1$ with extremely good accuracy, corresponding to missing-mass resolution $\Delta M \simeq 250 \mathrm{MeV}$. The experimental proposal is focused on the searches for the Higgs boson, possible manifestations of the physics beyond the Standard Model as well as on unique studies

of some subtle aspects of QCD dynamics. It is expected that, in association with the high $x_{p}$ protons and antiprotons, a central system with mass up to about $200 \mathrm{GeV}$ can be produced. To perform such challenging measurements the outgoing $p$ and $\bar{p}$ must be detected in roman pots, or microstations as proposed for an extension of ATLAS [11].

To ascertain whether a Higgs signal can be seen, it is crucial to evaluate the background. Recall that the inclusive search for an intermediate mass Higgs, that is $p p$ or $p \bar{p} \rightarrow H X$ with $H \rightarrow b \bar{b}$, has an extremely small signal-to-background ratio, which makes this process impossible to observe. If we specialize to double-diffractive $H \rightarrow b \bar{b}$ production, the background is suppressed [12] (see also Section 3).

\section{Double-diffractive hard production processes}

Here we are going to present the estimates of the cross-sections for high energy processes of the type

$$
p p \rightarrow p+M+p,
$$




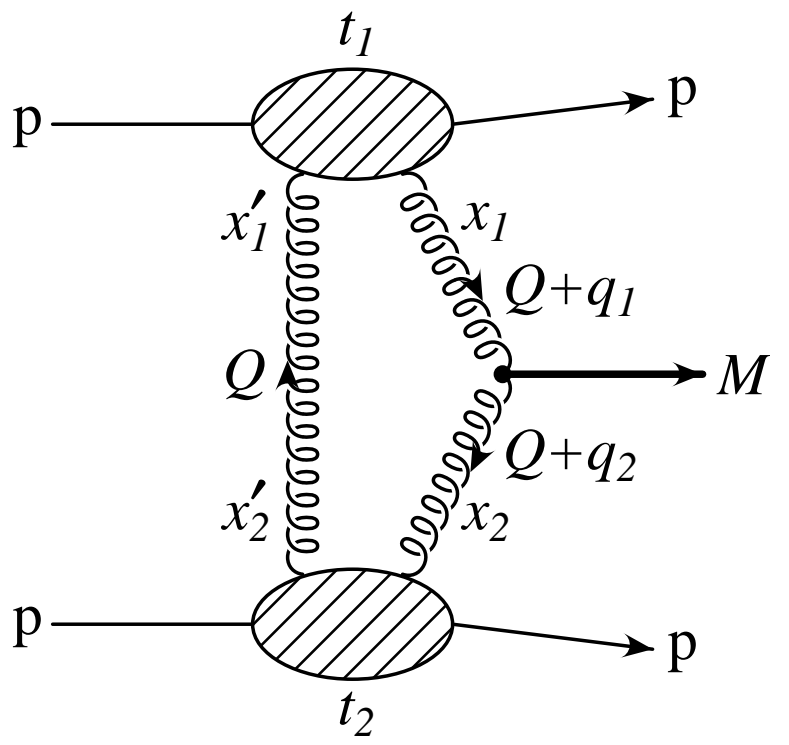

Figure 1: Schematic diagram of double-diffractive production of a system of invariant mass $M$, that is the process $p p \rightarrow p+M+p$.

and similarly for $p \bar{p}$, where a 'plus' signs indicates the presence of a large rapidity gap. To be precise, we calculate the rate for the double-diffractive exclusive production of a system of large invariant mass $M$, say a Higgs boson. Our discussion below will be focused on the case of an intermediate mass Higgs which dominantly decays into the $b \bar{b}$ final state. From the outset we would like to make it clear that today there is no consensus within the community regarding the evaluation of the double-diffractive production cross sections, see [13, 14]. The literature shows a wide range of predictions varying by many orders of magnitude円. As in Refs. 4, 6, 7, 8, 12, 15], we adopt the two-gluon exchange picture of the Pomeron, where the amplitude for the double-diffractive process is shown in Fig. 1. Here the hard subprocess $g g \rightarrow M$ is initiated by gluon-gluon fusion and an additional relatively soft $t$-channel gluon is needed to screen the colour flow across the rapidity gap intervals.

One major difference between the various theoretical approaches concerns the specification of the exchanged gluons. Either non-perturbative gluons are used in which the propagator is modified so as to reproduce the total cross section [4, 8], or a perturbative QCD estimate is made [7] using an unintegrated, skewed gluon density that is determined from the conventional gluon obtained in global parton analyses. In this respect, it has been emphasized [16] (see also [6, 7] that the non-perturbative normalisation based on the value of the elastic or total cross section fixes the diagonal gluon density at $\hat{x} \sim \ell_{T} / \sqrt{s}$ where the transverse momentum $\ell_{T}$ is

\footnotetext{
${ }^{1}$ The range of values for exclusive process (11) can be found in $6,6,9,10,12,14$.
} 
small, namely $\ell_{T}<1 \mathrm{GeV}$. Thus the value of $\hat{x}$ is even smaller than

$$
x^{\prime} \approx \frac{Q_{T}}{\sqrt{s}} \ll x \approx \frac{M}{\sqrt{s}},
$$

where the variables are defined in Fig. 1. However, the gluon density grows as $x \rightarrow 0$ and so the use of a non-perturbative normalisation will lead to an overestimation of double-diffractive cross sections.

Of course the fusion of the two energetic gluons into a high mass state, as shown in Fig. 1, is generally accompanied by the emission of soft gluons which may populate the rapidity gaps. The basic mechanism which suppresses this effect is the additional $t$-channel gluon of Fig. 1 . It screens the colour, but does not couple to the produced state of mass $M$. It has typical values of $Q_{T}$ which are much smaller than $M$, but which are large enough (for sufficiently large $M$ ) to screen soft gluon emission and to justify the applicability of perturbative QCD.

Another crucial numerical difference between the approaches concerns the size of the socalled survival probability of rapidity gaps, $W$, which symbolically can be written as

$$
W=S^{2} T^{2} .
$$

$S^{2}$ is the probability that the gaps are not filled by secondary particles generated by soft rescattering, see for instance [2, 3, 5, 6, 7, 14, 17, 18, 19]. The second factor, $T^{2}$, is the price to pay for not having gluon radiation in the hard subprocess $g g \rightarrow M$. The estimates of the survival factor $S^{2}$ strongly depend on the models for soft hadron rescattering, which causes the main uncertainty in evaluation of the double-diffractive cross section. In [7] for $M_{H}=120 \mathrm{GeV}$ at the LHC we tabulated our results for an optimistic estimate $S^{2}=0.1$, while our detailed recent calculations [18] yield a lower value, $S^{2}=0.02$. However it has been pointed out [19, 7] that it is possible to check the value of $S^{2}$ by observing double-diffractive dijet production?. This process is driven by the same dynamics, and has a higher cross section, so a comparison of the measurements with the predictions can determine $S^{2}$. The recent CDF data for diffractive dijet production, reported at this Workshop [21, 22], appear to be consistent with our determination of $S^{2}$ in Ref. [19]. This is clear evidence in favour of the strong suppression due to the low survival probability of the rapidity gaps.

The $p^{(} \bar{p}^{)} \rightarrow p+H+{ }^{(} \bar{p}^{)}$cross section, corresponding to the basic mechanism shown in Fig. 1, recently has been calculated to single log accuracy [7]. The amplitude is

$$
\mathcal{M}=A \pi^{3} \int \frac{d^{2} Q_{T}}{Q_{T}^{4}} f_{g}\left(x_{1}, x_{1}^{\prime}, Q_{T}^{2}, M_{H}^{2} / 4\right) f_{g}\left(x_{2}, x_{2}^{\prime}, Q_{T}^{2}, M_{H}^{2} / 4\right),
$$

where the $g g \rightarrow H$ vertex factor $A^{2}$ is given by (9) below, and the unintegrated skewed gluon densities are related to the conventional distributions by

$$
f_{g}\left(x, x^{\prime}, Q_{T}^{2}, M_{H}^{2} / 4\right)=R_{g} \frac{\partial}{\partial \ln Q_{T}^{2}}\left[\sqrt{T\left(Q_{T}, M_{H} / 2\right)} x g\left(x, Q_{T}^{2}\right)\right] .
$$

\footnotetext{
${ }^{2} \mathrm{~A}$ promising idea of probing the gap survival factor in the $W W$-fusion events with a rapidity gap on either proton side was advocated in Ref. [20]. For this purpose it was proposed there to measure the $Z^{0}$-production rate with the rapidity gap signature.
} 
The factor $R_{g}$ is the ratio of the skewed $x^{\prime} \ll x$ integrated gluon distribution to the conventional one [23]. $R_{g} \simeq 1.2(1.4)$ at LHC (Tevatron) energies. The bremsstrahlung survival probability $T^{2}$ is given by

$$
T\left(Q_{T}, \mu\right)=\exp \left(-\int_{Q_{T}^{2}}^{\mu^{2}} \frac{d k_{T}^{2}}{k_{T}^{2}} \frac{\alpha_{S}\left(k_{T}^{2}\right)}{2 \pi} \int_{0}^{1-k_{T} / \mu} d z\left[z P_{g g}(z)+\sum_{q} P_{q g}(z)\right]\right) .
$$

Note, that the factor $\sqrt{T}$ arises in (5) because the survival probability is only relevant to the hard gluon exchanges in Fig. 1. The origin of the factor $1 / Q_{T}^{4}$ in the integrand in (4) reflects the fact that the production is mediated by the 'fusion' of two colourless dipoles of size $d^{2} \sim 1 / Q_{T}^{2}$. Due to the presence of this factor it is argued (see e.g. [9]) that a perturbative treatment of process $(\mathbb{I})$ is inappropriate. However, it is just the Sudakov suppression factor $T$ in the integrand, which makes the integration infrared stable and hence the perturbative predictions reliables. The saddle points of the integral are located near $Q_{T}^{2}=3.2(1.5) \mathrm{GeV}^{2}$ at LHC (Tevatron) energies.

The bremsstrahlung survival factor $T$ determines the probability not to emit the bremsstrahlung gluons in the interval $Q_{T} \lesssim k_{T} \lesssim M_{H} / 2$. The upper bound of $k_{T}$ is clear, and the lower bound occurs because there is destructive interference of the amplitude in which the bremsstrahlung gluon is emitted from a "hard" gluon with that in which it is emitted from the screening gluon. That is there is no emission when $\lambda \simeq 1 / k_{T}$ is larger than the separation $d \sim 1 / Q_{T}$ of the two $t$-channel gluons in the transverse plane, since then they act effectively as a colour-singlet system. The neglect of such an important dampening factor in practically all theoretical papers on the double-diffractive Higgs or dijet production reminds one of the attempt to force two camels to go through the eye of a needle. The size of the eye is given by $1 / M$, while the camel's height is regulated by the position of the saddle point, $1 / Q_{T}$.

The amplitude (近) corresponds to the exclusive process (1). The modification for the inclusive process

$$
p p \rightarrow X+M+Y
$$

is given in [6, 0, 15], where it was found that the event rate is much larger. However, in the inclusive case the large multiplicity of secondaries poses an additional problem in identifying the Higgs boson.

\section{Signal-to-background ratio for double-diffractive Higgs production}

In order to use the 'missing-mass' method to search for an intermediate mass Higgs boson, via the $H \rightarrow b \bar{b}$ decay mode, we have to estimate the QCD background which arises from

\footnotetext{
${ }^{3}$ Moreover, the effective anomalous dimension, $\gamma$, of the gluon distribution $\left(x g\left(x, Q_{T}^{2}\right) \sim\left(Q_{T}^{2}\right)^{\gamma}\right)$ additionally suppresses the contribution from the low $Q_{T}^{2}$ domain [6].

${ }^{4}$ The only exceptions are our results in Refs. [6, 6, 12] and the evaluation presented in 8, 9. However there is a clear difference in the estimate of the survival factor $T^{2}$ even between these two groups. The calculation in [6, 7 [12 yields a significantly lower value of $T^{2}$ than that advocated in [8, 9].
} 
the production of a pair of jets with invariant mass about $M_{H}$, see Ref. [12 for details. The good news is that the signal-to-background ratio does not depend on the uncertainty in the soft survival factor $S^{2}$, and is given just by the ratio of the corresponding $g g \rightarrow H \rightarrow b \bar{b}$ and $g g \rightarrow b \bar{b}$ subprocesses.

We begin by assuming that the $b$ jets are not tagged. Then the main background is the double-diffractive colour-singlet production of a pair of high $E_{T}$ gluons with rapidities $\eta_{1}$ and $\eta_{2}$. The $g g \rightarrow g g$ subprocess cross section is [15, 24]

$$
\frac{d \hat{\sigma}}{d^{2} p_{T}}=\frac{9 \alpha_{S}^{2}}{8 p_{T}^{6}}\left(\frac{M^{4}}{4 p_{T}^{4}}-\frac{M^{2}}{p_{T}^{2}}\right)^{-\frac{1}{2}} \frac{d M^{2}}{d(\Delta \eta)}
$$

where $M$ is the invariant mass of the dijet system, $p_{T}$ is the transverse momentum of the jets, and $\Delta \eta=\left|\eta_{1}-\eta_{2}\right|$ is the jet rapidity difference. The background (8) should be compared to the double-diffractive $g g \rightarrow H$ signal

$$
\frac{A^{2}}{4}=\frac{\sqrt{2}}{36 \pi^{2}} G_{F} \alpha_{S}^{2}
$$

First of all, in order to reduce the background we impose a jet $E_{T}$-cut. For instance, if we trigger on events containing a pair of jets with angles $\theta>60^{\circ}$ from the proton direction in the Higgs rest frame, then we only eliminate one half of the signal, whereas the background dijet cross section is reduced to

$$
\frac{d \hat{\sigma}}{d M^{2}}=9.7 \frac{9 \alpha_{S}^{2}}{8 M^{4}}
$$

With a common scale for the coupling $\alpha_{S}$, and neglecting the NLO corrections, we obtain a signal-to-background ratio

$$
\frac{S}{B_{g g}}=\left(4.3 \times 10^{-3}\right) \operatorname{Br}(H \rightarrow b \bar{b})\left(\frac{M}{100 \mathrm{GeV}}\right)^{3}\left(\frac{250 \mathrm{MeV}}{\Delta M}\right)
$$

If $M_{H}=120 \mathrm{GeV}$, the ratio $S / B_{g g} \sim 5 \times 10^{-3}$. This is too small for the above approach to provide a viable signal for the Higgs boson. However the situation is greatly improved if we are able to identify the $b$ and $\bar{b}$ jets. If we assume that there is only a $1 \%$ chance to misidentify a gluon jet as a $b$ jet, then tagging both the $b$ and $\bar{b}$ jets will suppress the gluon background by $10^{4}$. In this case only the true $b \bar{b}$ background may pose a problem.

A remarkable advantage of the double-rapidity-gap signature for the $H \rightarrow b \bar{b}$ events is that here the $H \rightarrow b \bar{b}$ signal $/ b \bar{b}$ background ratio is strongly enhanced due to the colour factors, gluon polarization selection and the spin $\frac{1}{2}$ nature of the quarks [12]. First, the background $b \bar{b}$-dijet rate is suppressed due to the absence of the colour-octet $b \bar{b}$-state. Thus, for $E_{T}^{2}<M^{2} / 4$ we have

$$
\frac{d \hat{\sigma}(g g \rightarrow b \bar{b})}{d \hat{\sigma}(g g \rightarrow g g)}<\frac{1}{4 \times 27}<10^{-2}
$$

Second, we emphasize that for the exclusive process the initial $g g$ state obeys special selection rules. Besides being a colour-singlet, for forward outgoing protons the projection of the total 
angular momentum is $J_{z}=0$ along the beam axis. On the other hand, the Born amplitude for light fermion pair production vanishes in this $J_{z}=0$ state, see, for example, 26]. This result follows from $P$ - and $T$-invariance and fermion helicity conservation of the $J_{z}=0$ amplitude [27]. Thus, if we were to neglect the $b$-quark mass $m_{b}$, then at leading order we would have no QCD $b \bar{b}$-dijet background at all. Even beyond LO, the interference between the signal and background amplitudes is negligibly small, since they have different helicity structure. Therefore the form of the peak, observed in double-diffractive exclusive $H \rightarrow b \bar{b}$ production, will not be affected by interference with $b \bar{b}$ jets produced by the pure QCD background process.

Of course, a non-vanishing $b \bar{b}$ background is predicted when we allow for the quark mass or if we emit an extra gluon. Nevertheless in the former case we still have an additional suppression to (12) of about a factor of $m_{b}^{2} / p_{T}^{2} \simeq 4 m_{b}^{2} / M_{H}^{2}<10^{-2}$, whereas in the latter case the extra suppression is about $\alpha_{S} / \pi \simeq 0.05$. Note that events containing the third (gluon) jet may be experimentally separated from Higgs decay, where the two jets are dominantly co-planar?. However, the price to pay for this separation is the further reduction of the signal caused by the Sudakov suppression of the final state radiation in the Higgs events [27].

Thus, the two-gluon fusion mechanism for hard production, illustrated in Fig. 1, provides a unique situation where the incoming hard two-gluon system is practically fully polarized with $J_{z}=0$. An explicit calculation [12], assuming $M_{H}=120 \mathrm{GeV}$ and imposing the $\theta>60^{\circ}$ cut of low $E_{T}$ jets, gives a signal-to-background ratio

$$
\frac{S}{B_{b \bar{b}}} \gtrsim 4\left(\frac{1 \mathrm{GeV}}{\Delta M}\right) .
$$

The signal is, thus, in excess of background even at mass resolution $\Delta M \sim 2 \mathrm{GeV}$, so the $b \bar{b}$ background should not be a problem?.

\section{Is the production rate of Higgs events with rapidity gaps large enough?}

While the predictions for the $S / B_{b \bar{b}}$-ratio look quite favourable for Higgs searching using the missing-mass method, the expected event rate casts a shadow on the feasibility of this approach (at least for experiments at the Tevatron). The cross section for exclusive double-diffractive Higgs production at Tevatron and LHC energies has been calculated by several authors [4, 6, 7, 8, 9]. In our recent analysis [18] the gap survival probability for the double-diffractive

\footnotetext{
${ }^{5}$ For light quark pair exclusive production $p+p \rightarrow p+q \bar{q}+p$, with forward outgoing protons, the cancellation was first observed by Pumplin [25, see also [24, 15].

${ }^{6}$ The situation here is similar to the signal-to-background ratio for intermediate mass Higgs production in polarised $\gamma \gamma$ collisions, which was studied in detail in [27, 28].

${ }^{7}$ Unfortunately the situation worsens for inclusive Higgs production, when the polarization arguments become redundant. In this case $S / B_{b \bar{b}}$ ratio is additionally suppressed by a factor $\sim 20-30$.

${ }^{8} \mathrm{~A}$ more complete set of references to related theoretical papers can be found in Ref. [10].
} 
process is estimated to be $S^{2}=0.05$ at $\sqrt{s}=2 \mathrm{TeV}$ and $S^{2}=0.02$ at $\sqrt{s}=14 \mathrm{TeV}$. If we incorporate these estimates into the perturbative QCD calculations of [7], we find

$$
\begin{aligned}
\sigma_{H} & =\sigma(p \bar{p} \rightarrow p+H+\bar{p}) \simeq 0.06 \mathrm{fb} \\
\sigma_{H} & =\sigma(p p \rightarrow p+H+p) \simeq 2.2 \mathrm{fb}
\end{aligned} \quad \text { at } \quad \begin{array}{r}
\sqrt{s}=2 \mathrm{TeV} \\
\sqrt{s}=14 \mathrm{TeV}
\end{array}
$$

for a Higgs boson of mass $120 \mathrm{GeV}$. These values are much lower than the expectations of other authors listed in [10. However, as we already mentioned, the recent CDF study of diffractive dijet production [21, 22], provides strong experimental evidence in favour of our pessimistic estimates of the survival factor $S^{2}$, see [19]. CDF [21, 22] have studied double-diffractive dijet production for jets with $E_{T}>7 \mathrm{GeV}$. They find an upper limit for the cross section, $\sigma$ (dijet) $<3.7 \mathrm{nb}$, as compared to our prediction of about $1 \mathrm{nb}$ 19. Using the dijet process as a monitor thus rules out the much larger predictions for $\sigma(p \bar{p} \rightarrow p+H+\bar{p})$ which exist in the literature. Unfortunately the prediction $\sigma_{H} \simeq 0.06 \mathrm{fb}$ of (14) means that Run II of the Tevatron, with an integrated luminosity of $\mathcal{L}=15 \mathrm{fb}^{-1}$, should yield less than an event. We should add that the double-diffractive Higgs search can also be made in the $\tau^{+} \tau^{-}$and $W W^{*}$ decay channels, but, due to the small branching ratios, the event rate is even less.

We emphasize that such a low expected signal cross section at the Tevatron just illustrates the high price to be paid for improving the $S / B_{b \bar{b}}$ ratio by selecting events with double rapidity gaps. On the other hand, a specific prediction of the perturbative approach is that the cross section $\sigma_{H}$ steeply grows with energy [6, 7] (c.f. (14) with (15)), in contrast to non-perturbative phenomenological models based on Ref. [曰. In fact, if we were to ignore the rapidity gap survival probability, $S^{2}$, then $\sigma_{H}$ would have increased by more than a factor of 100 in going from $\sqrt{s}=2 \mathrm{TeV}$ to $\sqrt{s}=14 \mathrm{TeV}$. However, at the larger energy, the probability to produce secondaries which populate the gaps increases, and as a result the $\sigma(p p \rightarrow p+H+p)$ increases only by a factor of 40 . Nevertheless, there is a real chance to observe double-diffractive Higgs production at the LHC, since both the cross section and the luminosity are much larger than at the Tevatron. Another test of our perturbative scenario is the behaviour of the dijet cross section with the jet $E_{T}$. Due to the $x$ dependence of the perturbative gluon, we predict a steeper fall off with increasing $E_{T}$ than the non-perturbative models [7].

For the inclusive production of a Higgs of mass $M_{H}=120 \mathrm{GeV}$ we expect, at the LHC energy, a cross section of the order of $40(4) \mathrm{fb}$, taking rapidity gaps $\Delta \eta=2(3)$ [7].

The double-diffractive dijet cross sections are much larger than those for Higgs production. For example, if we take a dijet bin of size $\delta E_{T}=10 \mathrm{GeV}$ for each jet and $\eta_{1}=\eta_{2}$ we obtain, for $E_{T}=50 \mathrm{GeV}$ jets at LHC energies,

$$
d \sigma_{\mathrm{excl}} /\left.d \eta\right|_{0} \simeq 40 \mathrm{pb}, \quad d \sigma_{\mathrm{incl}} /\left.d \eta\right|_{0} \simeq 250 \mathrm{pb}
$$

where $\eta \equiv\left(\eta_{1}+\eta_{2}\right) / 2$. The rapidity gaps are taken to be $\Delta \eta($ veto $)=\left(\eta_{\min }, \eta_{\max }\right)=(2,4)$ for the inclusive case (see [15] for the definition of the kinematics). Such a high event rate and the remarkable purity of the di-gluon system, that is generated in the exclusive double-diffractive 
production process, provides a unique environment to make a detailed examination of high energy gluon jets'?. Indeed, we may speak here of a 'gluon factory' 12.

Let us finally comment on the soft suppression factor $S^{2}$, which causes the main uncertainty in various calculations of the rate of rapidity gap events and, thus, is the 'Achilles heel' of the evaluation of the Higgs production signal in the exclusive and inclusive processes of (11) and (17) respectively. This factor depends sensitively on the spatial distributions of partons inside the proton, and thus, is closely related to the whole diffractive part of the $S$-matrix, see Ref. [29] for details. For example, the survival probability for central Higgs production by $W W$ fusion, with large rapidity gaps on either side, is expected to be larger than that for the double-Pomeron exchange [7, 29]. Recall that a quantitative probe of the suppression factor $S^{2}$ can be achieved either by measurements of central $Z$ production [20] or of dijet production.

An instructive example is Higgs boson production by the $\gamma \gamma \rightarrow H$ fusion subprocess [30]. This process takes place at very large impact parameters, where the corresponding gap survival probabilities are $S^{2}=1$ [7, 31]. $\sigma(\gamma \gamma \rightarrow H)$ is estimated to be about $0.03 \mathrm{fb}$ at $\sqrt{s}=2 \mathrm{TeV}$ and $0.3 \mathrm{fb}$ at $\sqrt{s}=14 \mathrm{TeV}$, which is comparable to our expectations (14), (15) for doublediffractive Higgs production. Note that the strong and electromagnetic contributions have negligible interference, because they occur at quite different values of the impact parameter.

As well known [32, the two-photon mechanism of Higgs production is especially interesting for heavy ion collisions where the photon flux scales with $Z^{2}$ ( $Z$ being the charge of the nucleus) [33. In the photon-photon case for $M_{H}=120 \mathrm{GeV}$ we expect a signal-to-background ratio $S / B_{b \bar{b}} \sim 1$, if the experimental resolution on the reconstruction of the $b \bar{b}$-invariant mass is $\sim 10 \mathrm{GeV}$, and if a cut $|\cos \theta|<0.7$ is imposed on the $b$ and $\bar{b}$ jets.

\section{Conclusions}

We have examined the possibility of performing a high resolution missing-mass search for the Higgs boson at the Tevatron, that is the process $p \bar{p} \rightarrow p+H+\bar{p}$ where a 'plus' denotes a large rapidity gap. Using a model-independent approach, we find that there is a huge QCD background arising from double-diffractive dijet production. A central detector to trigger on large $E_{T}$ jets is essential. Even so, the signal-to-background ratio is too small for a viable 'missing-mass' Higgs search. The situation is much improved if we identify the $b$ and $\bar{b}$ jets. The $g g \rightarrow H \rightarrow b \bar{b}$ signal is now in excess of the QCD $g g \rightarrow b \bar{b}$ background, even for a mass resolution of $\Delta M \sim 2 \mathrm{GeV}$. The only problem is that, when proper account is taken of the survival probability of the rapidity gaps, the $p \bar{p} \rightarrow p+H+\bar{p}$ event rate is too small at the Tevatron. Recall that the experimental limit on the cross section for double-diffractive dijet production confirms the small predicted rates. Nevertheless, there is a real chance to observe

\footnotetext{
${ }^{9}$ In double-diffractive exclusive high- $E_{T}$ dijet events the jets appear to be pure gluon ones at the level about 3000:1. Moreover, after an appropriate selection of the two-jet configuration and the removal of the $b \bar{b}$ contamination by tagging, the sample may become (at least) an order of magnitude purer.
} 
double-diffractive Higgs production at the LHC, since both the cross section and the luminosity are much larger than at the Tevatron.

The rather pessimistic expectations of the missing-mass Higgs search at the Tevatron are, however, compensated by a by-product of the double-diffractive proposal. The doublediffractive production of dijets offers a unique gluon factory, generating huge numbers of essentially pure gluon jets from a colour-singlet state in an exceptionally clean environment.

\section{Acknowledgements}

We thank M. Albrow, D. Goulianos, A.B. Kaidalov, R. Orava, A. Rostovtsev and W.J. Stirling for useful discussions. VAK thanks The Leverhulme Trust for a Fellowship. This work was also supported by PPARC, the Russian Fund for Fundamental Research (98-01-17629) and the EU Framework TMR programme, contract FMRX-CT98-0194 (DG-12-MIHT). 


\section{References}

[1] Yu.L. Dokshitzer, V.A. Khoze and S.I. Troyan, Sov. J. Nucl. Phys. 46 (1987) 712.

[2] Yu.L. Dokshitzer, V.A. Khoze and T. Sjöstrand, Phys. Lett. B274 (1992) 116.

[3] J.D. Bjorken, Int. J. Mod. Phys. A7 (1992) 4189; Phys. Rev. D47 (1993) 101.

[4] A. Bialas and P.V. Landshoff, Phys. Lett. B256 (1991) 540.

[5] R.S. Fletcher and T. Stelzer, Phys. Rev. D48 (1993) 5162 and references therein.

[6] V.A. Khoze, A.D. Martin and M.G. Ryskin, Phys. Lett. B401 (1997) 330.

[7] V.A. Khoze, A.D. Martin and M.G. Ryskin, Eur. Phys. J. C14 (2000) 525.

[8] E.M. Levin, hep-ph/9912403 and references therein.

[9] D. Kharzeev and E.M. Levin, hep-ph/0005311.

[10] M.G. Albrow and A. Rostovtsev, hep-ph/0009336.

[11] V. Nomokonov, these proceedings.

[12] V.A. Khoze, A.D. Martin and M.G. Ryskin, hep-ph/0011393 and references therein.

[13] P.V. Landshoff, these proceedings.

[14] M.M. Block, these proceedings.

[15] V.A. Khoze, A.D. Martin and M.G. Ryskin, Phys. Rev. D56 (1997) 5867.

[16] A. Berera, Phys. Rev. D62 (2000) 014015.

[17] E. Gotsman, E.M. Levin and U. Maor, Phys. Lett. B353 (1995) 526; Phys. Rev. D60 (1999) 094011 and references therein.

[18] V.A. Khoze, A.D. Martin and M.G. Ryskin, Eur. Phys. J. C18 (2000) 167.

[19] V.A. Khoze, A.D. Martin and M.G. Ryskin, hep-ph/0006005; hep-ph/0007083, Phys. Lett. B (in press).

[20] H. Chehime and D. Zeppenfeld, Phys. Rev. D47 (1993) 3898.

[21] G. Snow, these proceedings.

[22] CDF Collaboration: T. Affolder et al., Phys. Rev. Lett. 85 (2000) 4215.

[23] A.G. Shuvaev, K.J. Golec-Biernat, A.D. Martin and M.G. Ryskin, Phys. Rev. D60 (1999) 014015. 
[24] A. Berera and J.C. Collins, Nucl. Phys. B474 (1996) 183.

[25] J. Pumplin, Phys. Rev. D52 (1995) 1477.

[26] K.A. Ispiryan, I.A. Nagorskaya, A.G. Oganesyan and V.A. Khoze, Sov. J. Nucl. Phys. 11 (1970) 712 .

[27] D.L. Borden, V.A. Khoze, W.J. Stirling and J. Ohnemus, Phys. Rev. D50 (1994) 4499;

V.S. Fadin, V.A. Khoze and A.D. Martin, Phys. Rev. D56 (1997) 484.

[28] M. Melles, W.J. Stirling and V.A. Khoze, Phys. Rev. D61 (2000) 054015.

[29] A.B. Kaidalov, V.A. Khoze, A.D. Martin and M.G. Ryskin, to be published.

[30] K. Piotrzkowski, these proceedings.

[31] V.A. Khoze, A.D. Martin, R. Orava and M.G. Ryskin, hep-ph/0010163, Eur. Phys. J. C (in press).

[32] E. Papageorgiu, Phys. Lett. B352 (1995) 394.

[33] L.D. Landau and E.M. Lifshitz, Sov. Phys. 6 (1934) 247. 